\title{
FLYING AND "AIR SICKNESS:"
}

Ax interesting lecture was delivered recently at a meeting of the Royal Aeronautical Society by Dr. C. A. Swan, followed by a discussion.

Dr. Swan emphasised the importance of the subject in view of the rapid approach of commercial aviation and increasing passenger transport. $\mathrm{He}$ purposely adopted the popular rather than the purely scientific standpoint, and gave an account of his personal experiences in the rarefied atmosphere of mountain altitudes. The physical and psychical facturs appear to be consideraibly intermingled: the material effects of a low atmospheric pressure are frequently accentuated by emotional disturbances. It seems that there is a definite altitude at which each individual's compensating mechanism begins to feel the strain; and individual capacity for readjustment is of greater importance than moderate changes in the environment. Muscular effort naturally increases symptoms of distress, which are rariously exhibited as dyspncea, cyanosis, vertigo, headache, tinnitus, vomiting, fainting, mental excitement, etc.

Simple remedies, such as black coffee, salol, and the old guide's remedy of oil of cinnamon on sugar, frequently effect relief, whilst the influence of posture is usually marked. The digestive organs and the higher nerve centres play a very large part.

Two types of altitude sickness have been described by Dr. Ravenhill, an acute form and a slow form, in which compensation is gradually lost. In the latter, cardiac or nervous symptoms may predominate. Compensation is attained by rise of blood pressure and by hyperpncea, both of which may be ascribed to a response of the medullary centres to lack of oxygen and psychical influences. In acclimatisation the acidity of the blood due to nonvolatile acids is increased, so that the respiratory centre becomes properly excited, although the alveolar carbon dioxide tension is low; in those who habitually dwell in mountain altitudes the red blood corpuscles are actually increased.

Dr. Swan cbserved that "staleness" in pilots is essentially due to fatigue of the mechanism for acclimatisation, and that rest is the first obvious point in treatment. Other causes of fatigue, such as eye-strain, dental sepsis, blocked nostrils, and mental worry should be eliminater? as far as possible. But the pilot flying under war conditions, driving a fast-climbing machine and repeatedly encountering alarming evidence of " hate," presents a very different problem from those who fly in civil life, although a remarkable proportion of war pilots came to grief through influence of altitude, whilst many flew successfully who, by all the rules of the game, should have been unfit for aviation in any circumstances.

In the discussion following Dr. Swan's address, General Brooke Popham laid stress on the importance of oxygen administration, and said that great benefit was derived from its use in France during the war. Dr. Stamm considered that the question of air-sickness will affect airship rather than aeroplane flying, and that the provision of oxygen affords no real difficulty. The chairman, Brigadier-General Bagnall Wild, attaches importance to a healthy digestion, and expressed the opinion that there need be no fear of suffering during passenger flights, apart from excessive altitudes (over 15,000 feet) and " stunting."

Aviation as a means of transport would appear to present no serious terrors in this direation to the healthy individual. Our systems are far more adaptable than we are too often encouraged to believe.

\section{The Problem of Venereal Disease.}

A GOOD deal of confusion has arisen in the public mind with regard to the distinction between the National Council for Combat:ing Venereal Diseases and the recentlyformed Society for the Prevention of Venereal Disease. Both bodies take their stand on common ground in desiring the reduction and final elimination of the terrible scourge of syphilis and gonorrhœa from our national life. But they approach and deal with the problem from entirely different points of view.

Basing their opinion on the firdings of the Royal Commission on Venereal Disease and the experience gained during the war, the National Council contends that the strongest weapons available are, first, the education of all classes and sections of the community as to the causes, nature, and results of those diseases, and, secondly, the earliest possible treatment of infected persons. They also recognise that immediate local cleanliness after exposure to the risk of infection will reduce that risk, but, unfortunately, it is by no means an infallible preventive. Where propaganda stating that it is so has taken place, the amount of venereal disease has materially increased-vide the incidence among the Australian troops, starting as it did at 130 per thousand and rising to 147 per thousand, as compared with the Br'tish troops' incidence of thirty-eight per thousand.

The Society for the Prevention of Venereal Disease consider that immediate self-disinfection is the only measure worthy of consideration from either a medical or social point of view. In this they are in disagreement with the opinion expressed by Lord Sandhurst on behalf of the Government during the debate in the House of Lords on December 10, when he stated that "even in circumstances specially favourable to instruction in the method proposed the risk of venereal disease is by no means certainly avo:ded.... How much more, then, would this be the case, and how far removed should we still be from secured immunity, with the civil population, in respect of whom the arrangements as to instruction and discipline adopted for the military would obviously be quite impossible?" The same view is held by an almost unanimous maiority of medical officers of health and clinic officers throughout the country. Many of those who support the policy of the Society for the Prevention of Venereal Disease bear illustrious names rightly honoured in the medical world-but they do not represent those medical men and women who have had long and intimate experience of the practical problems involved in the successful prevention and treatment of venereal disease. The Society for the Prevention of Venereal Disease is therefore completely at variance with the National Council in regarding immediate self-disinfection as the one and only practical measure by which venereal disease can be exterminated. The National Council considers it but one of several weapons to be added to the armoury of education and prompt treatment. 